\title{
Asymmetrical excitation of surface plasmon polaritons on blazed gratings at normal incidence
}

\author{
Benfeng Bai, ${ }^{1 *}$ Xiangfeng Meng, ${ }^{1,2}$ Janne Laukkanen, ${ }^{1}$ Tristan Sfez, ${ }^{3}$ Libo Yu, ${ }^{3}$ Wataru Nakagawa, ${ }^{4}$ \\ Hans Peter Herzig, ${ }^{3}$ Lifeng $\mathrm{Li}^{2}{ }^{2}$ and Jari Turunen ${ }^{1}$ \\ ${ }^{1}$ Department of Physics and Mathematics, University of Joensuu, P.O. Box 111, FI-80101 Joensuu, Finland \\ ${ }^{2}$ Department of Precision Instruments, Tsinghua University, Beijing 100084, China \\ ${ }^{3}$ Ecole Polytechnique Fédérale de Lausanne (EPFL), Institute of Microengineering (IMT-OPT), Breguet 2, 2000 Neuchâtel, Switzerland \\ ${ }^{4}$ Department of Electrical and Computer Engineering, Montana State University, P.O. Box 173780, Bozeman, \\ Montana 59717-3780, USA
}

(Received 20 April 2009; revised manuscript received 12 June 2009; published 9 July 2009)

\begin{abstract}
We present results of numerical simulations and preliminary experiments to investigate and characterize the effect of asymmetrical coupling of normally incident light to surface plasmon polaritons (SPPs) on metallic blazed gratings. Two types of blazed gratings are investigated, a two-dimensional (2D) area-coded binary grating and a one-dimensional (1D) slanted sinusoidal grating. The 2D blazed grating, which can be fabricated with standard $e$-beam lithography, is shown to have the same ability as the classical 1D blazed grating to enhance the strength of the $-1 \mathrm{st}(+1 \mathrm{st})$ evanescent order over the $+1 \mathrm{st}(-1 \mathrm{st})$ counterpart, which leads to the asymmetrical excitation of two counterpropagating SPP modes on the grating surface. The 1D blazed grating, as a reference, is also studied experimentally to verify the previous theoretical predictions. In our first experiments, the observed asymmetrical coupling effect is relatively weak compared with the optimal designs due to many practical limitations. However, good agreement between theory and experiment has been obtained, and physical insight concerning the observed SPP coupling phenomena has been gained. Further measures to realize stronger asymmetrical excitation of SPPs on blazed gratings at normal incidence are discussed.
\end{abstract}

DOI: 10.1103/PhysRevB.80.035407

PACS number(s): 73.20.Mf, 78.66.Bz, 42.79.Dj

\section{INTRODUCTION}

The excitation and manipulation of surface plasmon polaritons (SPPs) is a vital issue for many nanophotonic applications based on light-matter interaction on metal surfaces, such as the proposed highly integrated photonic circuits. ${ }^{1}$ As one of several alternatives for the excitation of SPPs, diffraction gratings have been widely used to couple incident light to SPPs on metal films. ${ }^{2}$ The directional excitation and waveguiding of SPPs can be achieved, for example, by oblique incidence of light on the grating, ${ }^{3,4}$ via the phase matching of one evanescent diffraction order to a SPP mode, or by back-side illumination of a nanoslit perforated in the metal film next to a periodic array of grooves that acts as a Bragg mirror to reflect the SPP wave away from the array. ${ }^{5}$

The oblique incidence condition is not always available in integrated optics applications, or additional complexity has to be included in the optical system design so that the incident angle can be precisely adjusted to meet the phasematching condition for SPP excitation. The slit-grating configuration, as a special design, is efficient for unidirectional SPP coupling. However, a simpler and more flexible coupling mechanism that allows unidirectional SPP excitation under normal incidence with either front- or back-side illumination is also welcome.

Recently, Bonod et al. proposed using slanted lamellar or sinusoidal gratings to launch unidirectionally propagating SPPs. ${ }^{6}$ The advantage is that the normally incident light can excite \pm 1 st evanescent orders with highly asymmetrical strengths due to the blazing effect of the slanted surfacerelief profile of the grating. By phase matching the momentum of the \pm 1 st evanescent orders with the in-plane plasmon wave vector, the incident energy can be asymmetrically coupled to the counterpropagating SPPs along the periodic direction. When the asymmetry effect is sufficiently large, even unidirectional SPP excitation can be attained.

Our present work is driven by two motivations. First, the fabrication of classical one-dimensional (1D) blazed gratings is more complicated than that of binary structures due to their slanted profiles. The asymmetrical profiles have to be manufactured by, e.g., direct laser-beam or ion-beam milling, a series of photolithographic processes that approximate the continuous surface profile by a staircase multilevel structure, or the inclined-angle reactive ion-beam etching with a binary grating mask. ${ }^{7}$ In addition to the complexity of the fabrication process (e.g., several masks have to be used to create a multilevel profile), the control of depth variation within a period, which is crucial for achieving the blazing effect, is rather difficult. In electron-beam lithography (EBL), controlling the in-plane dimensions of a binary structure is much easier than modulating its depth. Based on this consideration, Kleemann et $a l .{ }^{8}$ proposed a novel type of binary-blazed gratings, called the blazed area-coded effective-medium structures (BLACES), to achieve the blazing effect through an effective index variation in a single layer by modulating the local filling factor of a binary two-dimensional (2D) grating pattern. Unlike the 1D slanted gratings or conventional binary-blazed gratings with high-aspect-ratio ridges or pillars, ${ }^{9}$ the BLACES have more reasonable dimensions and are easier to fabricate with, e.g., standard EBL. ${ }^{10} \mathrm{We}$ thus propose the use of BLACES as an alternative for the asymmetrical coupling of SPPs.

Second, the asymmetrical coupling of SPPs on blazed gratings, although theoretically predicted to be large,${ }^{6}$ has not been corroborated experimentally so far. In this work, we carry out preliminary experiments to characterize the effect on blazed grating samples, including both BLACES and the 
slanted gratings. Although the observed asymmetrical coupling effect is relatively weak as the samples do not fit the optimal designs due to many practical restrictions in our experiments, the characterization results still provide useful information to validate the theoretical predictions.

The paper is organized as follows. In Sec. II, the fundamental principle of asymmetrical excitation of SPPs by blazed gratings is illustrated. Then two types of blazed gratings, the BLACES and the 1D slanted gratings, are thoroughly studied in Secs. III and IV, respectively. Each includes theoretical analysis/design, sample preparation, and optical characterization. Discussions and conclusions of the whole work are presented in Sec. V.

\section{FUNDAMENTAL PRINCIPLE}

Surface plasmons are electron oscillations near a metal surface stemming from the broken translational invariance in the direction normal to the surface. A combined excitation consisting of a surface plasmon and a photon is called a SPP. ${ }^{11}$ The dispersion relation of a SPP on a smooth metal surface is given by ${ }^{2}$

$$
k_{\mathrm{SPP}}=k_{0} \sqrt{\frac{\epsilon_{\mathrm{met}} \epsilon_{\mathrm{die}}}{\epsilon_{\mathrm{met}}+\epsilon_{\mathrm{die}}}},
$$

where $k_{\mathrm{SPP}}$ is the modulus of the SPP wave vector $\mathbf{k}_{\mathrm{SPP}}, k_{0}$ $=2 \pi / \lambda_{0}$ is the light wave number in vacuum, and $\epsilon_{\text {met }}$ and $\epsilon_{\mathrm{die}}$ are permittivities of the metal and the adjacent dielectric, respectively.

Since the SPP dispersion curve always lies on the right side of the light line without crossing it (see, for example, Ref. 2), a SPP cannot couple to a radiative mode and, conversely, cannot be directly excited by light propagating in the dielectric. However, if a grating with period $d$ is introduced, momentum conservation can be satisfied through the $m$ th evanescent diffraction order by ${ }^{2}$

$$
\mathbf{k}_{\mathrm{SPP}}=\mathbf{k}_{\|}^{\mathrm{inc}}+m K \hat{\mathbf{x}},
$$

where $\mathbf{k}_{\|}^{\text {inc }}$ is the in-plane wave vector of the incident light and $K=2 \pi / d$. Specifically, if the grating is mounted under normal incidence (so that $\mathbf{k}_{\|}^{\text {inc }}=0$ ) and the SPP is to be excited by the \pm 1 st orders, Eq. (2) is reduced to

$$
\mathbf{k}_{\mathrm{SPP}}= \pm K \hat{\mathbf{x}} \text {. }
$$

Therefore, according to Eqs. (1) and (3), we can estimate, e.g., the excitation light wavelength $\lambda_{0}$, the SPP wavelength $\Lambda_{\mathrm{SPP}}=2 \pi / k_{\mathrm{SPP}}$ on the metal surface, or the grating period $d$, to obtain a preliminary guess of the parameters for a specific design.

The two types of blazed gratings studied in this work, a BLACES and a slanted sinusoidal grating, are depicted in Fig. 1. The BLACES consists of a planar gold film perforated with an array of isosceles triangular holes and a substrate with permittivity $\epsilon_{\text {sub }}$. The gold film thickness $h$, the array periods $d$ and $p$ (in $x$ and $z$ directions, respectively), and the triangle dimensions $a$ and $b$ are given in Fig. 1(a). Since the transverse period $p$ is much smaller than the incident wavelength $\lambda_{0}$ and the blaze period $d$, all diffraction
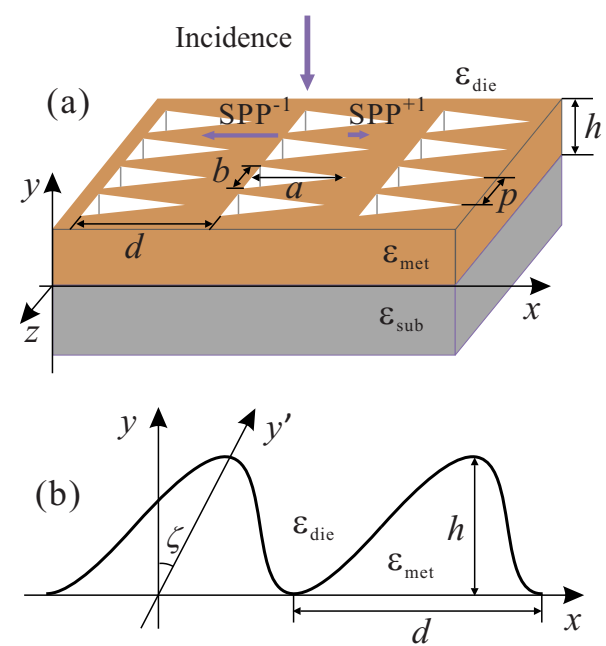

FIG. 1. (Color online) Geometries of (a) a BLACES and (b) a slanted sinusoidal grating. $\mathrm{SPP}^{ \pm 1}$ refer to the two SPP modes asymmetrically excited by the \pm 1 st evanescent orders at normal incidence (the lengths of arrows show the relative strength).

orders, except for the zeroth order, are evanescent in the $z$ direction. Therefore, the diffraction spectrum of this $2 \mathrm{D}$ grating is similar to that of a 1D grating, with the blazing effect induced by the local effective refractive index modulation within each period along the $x$ direction. ${ }^{8,10}$ The classical slanted grating in Fig. 1(b), however, achieves the blazing effect with the aid of its asymmetrical surface-relief profile.

By blazing, the diffraction efficiencies of the \pm 1 st reflected orders (in the $x$ direction) can be highly asymmetric, i.e., most of the diffracted light is redirected into a single order. In such circumstances, if the grating period $d$ decreases so that the \pm 1 st orders turn from propagating to evanescent and match the SPP momentum via Eq. (3), the excited SPP modes propagating in $+x$ and $-x$ directions could have asymmetric strengths as the incident energy is transferred to the SPPs via the \pm 1 st orders. This is the fundamental principle for the asymmetrical excitation of SPPs on blazed gratings.

\section{BLAZED AREA-CODED EFFECTIVE MEDIUM STRUCTURE}

\section{A. Theoretical and numerical studies}

The Fourier modal method (FMM), reformulated with a group-theoretic approach for crossed gratings with one reflection symmetry, ${ }^{12}$ is used to simulate the optical response of the BLACES. The normally incident plane wave is assumed to be $x$ polarized because the SPPs can only be excited by TM-polarized light, ${ }^{2}$ which in our case means that the incident electric field is parallel to the $x$ axis.

For the 1D slanted grating, due to the split of TE and TM fields, the excited field is still TM polarized. For crossed gratings, there are usually no isolated TE and TM solutions, i.e., all six field components are coupled to each other. However, for the BLACES shown in Fig. 1(a), due to the symmetries of both the structure and the incident condition, only 
the field of one symmetry mode can be excited, which is expressed as ${ }^{12}$

$$
H_{x}(\mathbf{r})=-H_{x}(\widetilde{\mathbf{r}}), \quad H_{y}(\mathbf{r})=-H_{y}(\widetilde{\mathbf{r}}), \quad H_{z}(\mathbf{r})=H_{z}(\widetilde{\mathbf{r}}),
$$

where $\mathbf{r}$ and $\tilde{\mathbf{r}}$ refer to two spatial points that are mirror symmetric with respect to the $O x y$ plane. In the free space above the grating, the diffracted field can be expressed by Rayleigh expansion

$$
H_{\sigma}(\mathbf{r})=\sum_{m, n} H_{\sigma m n} \exp \left[i\left(\alpha_{m} x+\beta_{n} z+\gamma_{m n} y\right)\right],
$$

where $\sigma=x, y, z$, and $\alpha_{m}, \beta_{n}$, and $\gamma_{m n}$ are the components of the wave vector of the $(m, n)$ th spatial harmonic. The property of the symmetry mode leads to ${ }^{12}$

$$
H_{x m 0}=H_{y m 0}=0 \text {, }
$$

meaning that the reflected field is quasi-TM polarized (i.e., $H_{z}$ is dominant) since the higher evanescent order amplitudes $H_{x m n}$ and $H_{y m n}$ with larger $n$ are relatively weak. Our numerical calculation will also verify this point. Therefore, to evaluate the asymmetrical coupling effect of the SPPs in $\pm x$ directions, we just need to calculate the amplitude ratio $r_{ \pm 1}$ $\equiv\left|H_{z(-1) 0} / H_{z(+1) 0}\right|$.

The simulation of the 2D metallic BLACES suffers from the slow convergence and heavy computation load when the traditional FMM is used. However, with the reformulated FMM, the computation time is reduced to $1 / 8$ and the convergence range is doubled, which facilitates our numerical optimization process.

The BLACES has five parameters, $d, p, a, b$, and $h$, to be optimized. As mentioned before, the blazing effect is induced by the gradient local effective dielectric constant of the grating layer, which for the $x$-polarized incidence can be expressed as ${ }^{10}$

$$
\epsilon_{\text {eff }}(x)=\epsilon_{\text {met }} f(x)+\epsilon_{\text {die }}[1-f(x)],
$$

where $f(x)$ is the local filling factor of metal at a given $x$. Therefore, to get the largest gradient of $\epsilon_{\text {eff }}$, we choose $a$ $=d$ and $b=p$ so that $\epsilon_{\text {eff }}$ can vary smoothly from $\epsilon_{\text {die }}$ to $\epsilon_{\mathrm{met}}$ with respect to $x$ within a period.

We choose gold as the metal and fused silica as the substrate material. The gold refractive index data used in the simulation are those measured on a $100-\mathrm{nm}$-thick evaporated gold film by ellipsometry, as the refractive index of thin metal film is considerably different from that of bulk metal. ${ }^{13}$ At wavelength $\lambda_{0}=633 \mathrm{~nm}$, the material permittivities are $\epsilon_{\mathrm{met}}=(0.1684+i 3.5337)^{2}, \epsilon_{\mathrm{sub}}=1.4569^{2}$, and $\epsilon_{\mathrm{die}}=1$ (for the cover medium air). Then Eqs. (1) and (3) can give us an estimate of the grating period $d \approx 607 \mathrm{~nm}$. To form the BLACES, the transverse period is chosen as $p \approx d / 2$, which is small enough and also feasible for EBL fabrication.

The optimal values of $d, p$, and $h$ are found by numerical optimization. Figure 2(a) shows the variation in $r_{ \pm 1}$ with respect to $p$ and $h$ with a preoptimized period $d=610 \mathrm{~nm}$. In the central white region, $r_{ \pm 1}$ can be higher than 500. In Fig. 2(b) we show the wavelength spectra of a BLACES with $p$ $=312.5 \mathrm{~nm}$ and $h=88 \mathrm{~nm}$ corresponding to point A in Fig. 2(a). The $H_{z( \pm 1) 0}$ and $H_{z 00}$ amplitudes, normalized with the incident magnetic field amplitude, as well as the total effi-
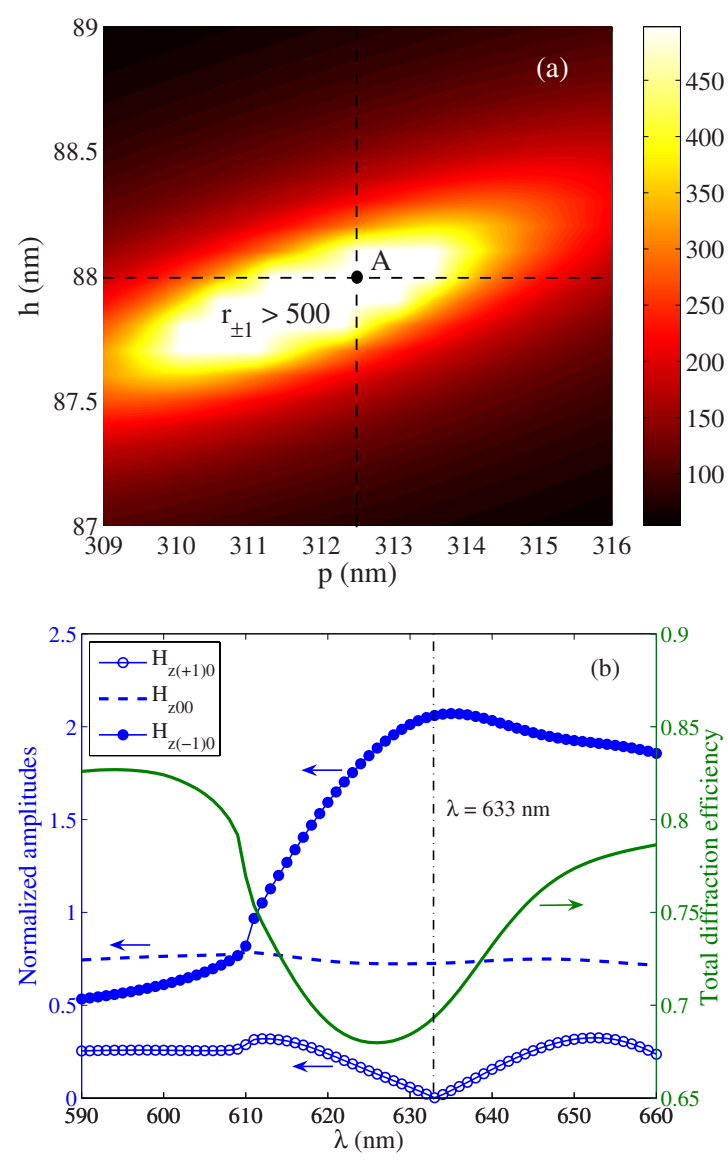

FIG. 2. (Color online) Optimization of a BLACES with period $d=610 \mathrm{~nm}$ under an $x$-polarized illumination with $\lambda_{0}=633 \mathrm{~nm}$. (a) Amplitude ratio $r_{ \pm 1}$ as a function of the transverse period $p$ and the grating layer thickness $h$. The white color shows the region with $r_{ \pm 1}>500$. (b) Wavelength spectra of the BLACES with $p$ $=312.5 \mathrm{~nm}$ and $h=88 \mathrm{~nm}$ corresponding to point A in (a). $H_{z( \pm 1) 0}$ and $H_{z 00}$ are amplitudes of the reflected \pm 1 st and zeroth orders normalized with the incident magnetic field amplitude. The total diffraction efficiency of all, reflected and transmitted, diffraction orders is also demonstrated.

ciency of all diffraction orders are demonstrated. Beyond $\lambda_{0}=610 \mathrm{~nm}$, the reflected $\pm 1 \mathrm{st}$ orders are evanescent. It is seen that the strengths of $H_{z( \pm 1) 0}$ are highly asymmetric in the vicinity of surface plasmon resonance, where there is a decrease in total diffraction efficiency because the incident energy is transferred more to the SPPs. Especially, at $\lambda_{0}$ $=633 \mathrm{~nm}, H_{z(+1) 0}$ is almost zero, leaving one SPP wave unidirectionally propagating in $-x$ direction.

To have an intuitive image of the coupling of incident light to the SPPs, we have calculated the energy flow and electromagnetic field distribution at various heights above the air-metal interface at $\lambda_{0}=633 \mathrm{~nm}$, as shown in Fig. 3. E and $\mathbf{H}$ are instantaneous electric and magnetic vectors, i.e., the real parts of the corresponding complex phasors $\widetilde{\mathbf{E}}$ and $\widetilde{\mathbf{H}}$, and the energy flow is expressed by the time-averaged Poynting vector $\langle\mathbf{S}\rangle=\frac{1}{2} \operatorname{Re}\left(\widetilde{\mathbf{E}} \times \widetilde{\mathbf{H}}^{*}\right)$. The far field in Fig. 3(a) clearly shows a uniform $x$-polarized plane wave with its energy flowing normally downward. When the wave approaches the grating surface, the energy is redirected to 

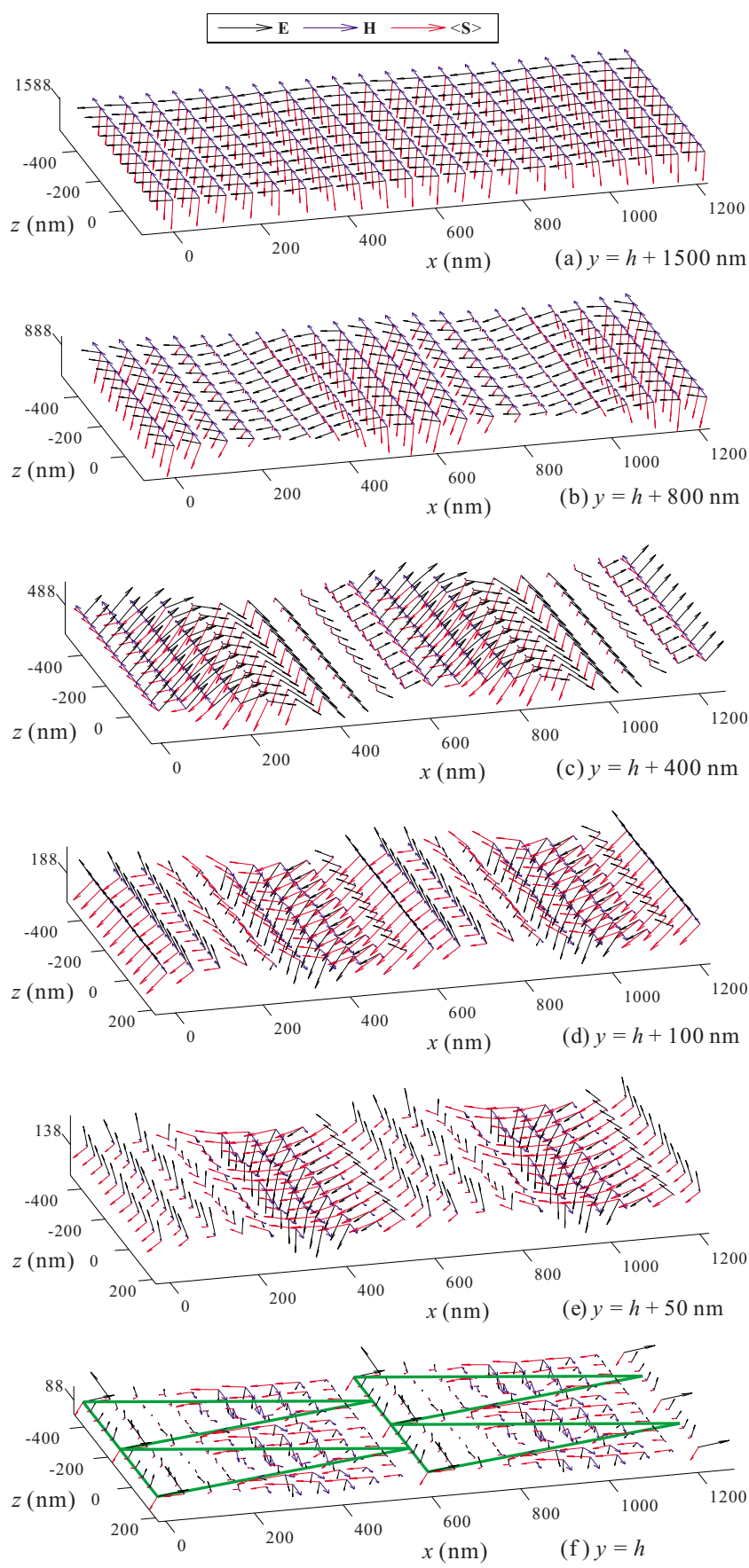

FIG. 3. (Color online) Distribution of the instantaneous $\mathbf{E}$ and $\mathbf{H}$ vectors as well as the time-averaged Poynting vector $\langle\mathbf{S}\rangle$ in the $x z$ planes $1500-0 \mathrm{~nm}$ above the air-metal interface, as shown in (a)(f). The arrow length is proportional to the magnitude of the vector.

propagate primarily in $-x$ direction. The dominant $H_{z}, E_{y}$, and $E_{x}$ components of the field and their spatial variations, shown in Figs. 3(d) and 3(e), unambiguously reveal the characteristics of a SPP field.

We should note that the calculated vectors are for the total field, but not for an individual wave, so that $\langle\mathbf{S}\rangle$ is actually a net energy flow of all propagating waves including the incident, reflected, and SPP (if in near field) ones. Therefore, even though we see a downward energy flow in Fig. 3(a), it does not mean that only one incident wave is propagating;

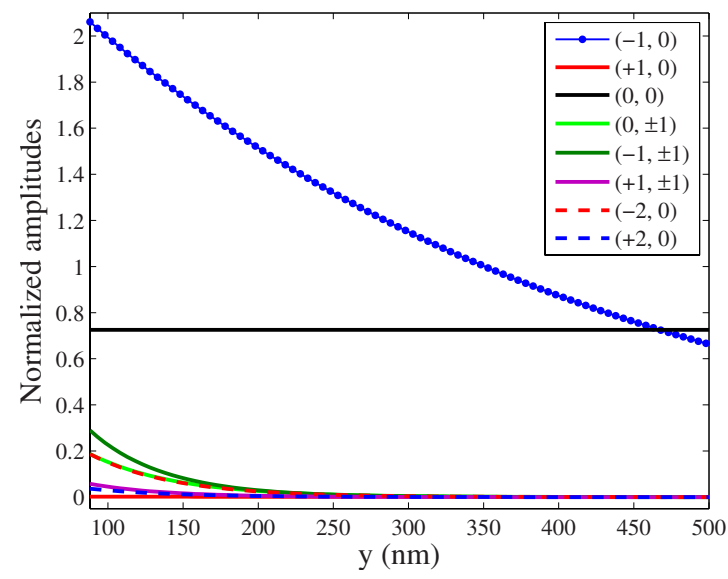

FIG. 4. (Color online) Amplitudes $\left|H_{z m n}(y)\right|$ of the $(m, n)$ th reflected orders normalized with the incident magnetic field amplitude (i.e., the incident magnetic field has unit amplitude). Note that the air-metal interface is at $y=h=88 \mathrm{~nm}$.

actually, the $(0,0)$ th reflected order is also involved, just that its $\langle\mathbf{S}\rangle$ is smaller than that of the incident field so that the net energy flow is still downward. Hence, we should understand Fig. 3 comprehensively by analyzing the field composition. Figure 4 shows the variation in amplitudes $\left|H_{z m n}(y)\right|$ $=\left|H_{z m n} \exp \left(i \gamma_{m n} y\right)\right|$ of the $(0,0)$ th propagating order and the other major evanescent orders in the near field. Obviously, the strength of the $(-1,0)$ th order is much stronger than the others and the higher evanescent orders also decay more rapidly. Therefore, the $(-1,0)$ th order makes major contribution to the near field and the energy flow can largely reflect the propagation of the SPP wave in $-x$ direction.

By comparing the results with those of Ref. 6, we can see that the BLACES can also realize the asymmetrical, or even unidirectional, excitation of SPPs under normal incidence. A potential concern is that the coupling efficiency of the incident light to SPPs on the designed BLACES is not as high as that on the $1 \mathrm{D}$ slanted gratings. ${ }^{6}$ This may be due to the fact that the planar metal surface is less efficient than the corrugated one to accumulate oscillating charges. Further study should be pursued to improve the coupling efficiency of SPPs on the BLACES. Despite this concern, the BLACES has been shown, in principle, to be a good alternative to the 1D slanted gratings to achieve highly asymmetrical excitation of SPPs, especially in view of its easier fabricability.

\section{B. Sample design and fabrication}

We have launched a preliminary experiment to characterize the asymmetrical excitation of SPPs on BLACES by near-field microscopy. The sample design is not ideal due to some practical restrictions. First, the illumination wavelength $\lambda_{0}$ has to be set around $1530 \mathrm{~nm}$ to match the light source of the scanning near-field optical microscope (SNOM) although the SPP excitation is less efficient in the infrared due to the increased metal conductivity. ${ }^{14,15}$ Moreover, only the airmetal interface can be characterized with an illumination from the back side of the sample so as to avoid mechanical interference of the light source with the near-field probe. The 


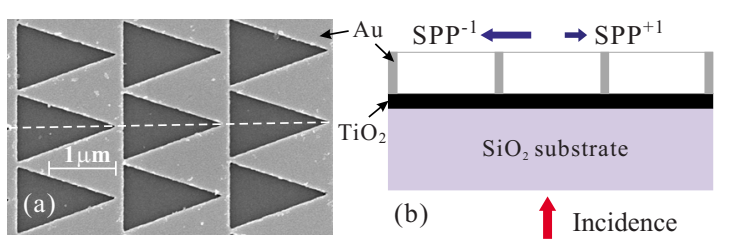

FIG. 5. (Color online) (a) Top-view SEM image of a fabricated BLACES sample. (b) Side-view schematic, corresponding to the dashed line in (a), of the sample in characterization with back-side illumination. $\mathrm{SPP}^{ \pm 1}$ have the same meaning as those in Fig. 1.

back side illumination is also disadvantageous for efficient asymmetrical excitation of SPPs on the front interface of the perforated metal film. Even though we have these limitations, it is still meaningful to characterize the effect (on the air-metal interface at the transmission side hereafter) experimentally and compare it with theoretical prediction.

The samples were fabricated by standard EBL and lift-off technique. A fused-silica substrate was first deposited with a 5-nm-thick $\mathrm{TiO}_{2}$ adhesion layer by thermal evaporation. Then a 120-nm-thick polymethyl methacrylate electronbeam resist layer was spin coated, which was covered by a 20-nm-thick conductive copper layer. The resist was patterned by a Vistec EBPG5000+ES high-resolution electronbeam writer. After the copper layer was removed and the resist developed, the sample was coated with a 40-nm-thick gold layer by thermal evaporation. The remaining resist and thus the gold on top of it were removed with acetone, and the sample was rinsed with isopropanol. The scanning electron microscope (SEM) image of one fabricated sample is shown in Fig. 5(a), whose structural parameters are approximately $d=1520 \mathrm{~nm}, p=1050 \mathrm{~nm}, a=1360 \mathrm{~nm}$, and $b=955 \mathrm{~nm}$.

The numerically calculated spectra (i.e., the normalized amplitudes of the transmitted orders as well as the total diffraction efficiency of all orders) of the sample are shown in Fig. 6, which indicate a surface plasmon resonance around wavelength of 1520-1540 nm. SPPs excited on the air-metal interface should be responsible for this resonance as the SPPexcitation wavelength estimated from Eqs. (1) and (3) is $\lambda_{0}$ $\approx 1527 \mathrm{~nm}$. By comparing Fig. 6 with Fig. 2(b), we can find a major difference that the +1 st-order amplitude is enhanced

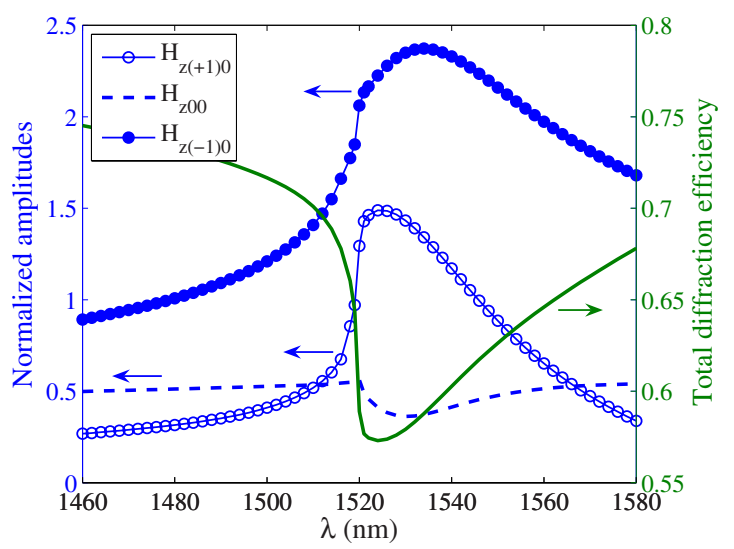

FIG. 6. (Color online) Numerical spectra of the fabricated BLACES sample. The notations are the same as those in Fig. 2 except that the amplitudes are for the transmitted orders.
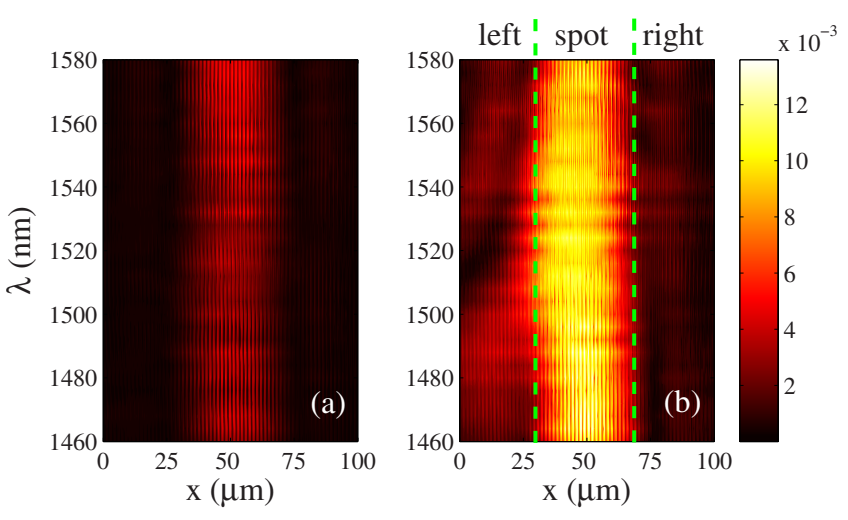

FIG. 7. (Color online) Near-field strength (square root of the field intensity) scanned in $x$ direction across the illumination spot. The scan length is $100 \mu \mathrm{m}$ and the wavelength is changed from 1460 to $1580 \mathrm{~nm}$ with a step of $4 \mathrm{~nm}$. The spot diameter is about $20 \mu \mathrm{m}$. (a) TE ( $z$-polarized) incidence. (b) TM ( $x$-polarized) incidence. The amplitude scale is in arbitrary units.

at the resonance but not suppressed although the -1 st-order amplitude is still enhanced. For this reason, the amplitude ratio $r_{ \pm}$of the transmitted \pm 1 st orders in this sample is not maximized at resonance and thereby does not lead to a large asymmetrical SPPs coupling effect on the air-metal interface. However, we should note that it may be possible to maximize $r_{ \pm}$also at transmission side by proper structural configuration because previous study ${ }^{16}$ has shown that the amplitudes of transmitted orders (at least the zeroth order) can appear as maxima, minima, or Fano-shaped resonances.

\section{Near-field characterization}

To characterize the effect experimentally, we employed a multiheterodyne SNOM (see Ref. 17 for the details of the characterization method and setup) to locally collect the near field by raster scanning the surface of the sample with a dielectric probe (Lovalite, $70 \mathrm{~nm} \mathrm{Al}$ coated, aperture of 200 $\mathrm{nm})$. The scan line was along the $x$ direction and the maximum scan length was $100 \mu \mathrm{m}$. The surface topography was obtained by means of shear-force detection. Based on the multiheterodyne inteferometric technique, the SNOM setup allowed simultaneous measurement of the amplitudes and phases of the optical near fields resulting from two orthogonally polarized illuminating beams during a single scan. In our experimental configuration, the sample was normally illuminated with a superposition of linearly TE (z-polarized) and TM ( $x$-polarized) beams generated by a tunable laser source (Agilent 81682A, spectral range of 1460-1580 nm). The illumination wavelength was changed with a step of 4 $\mathrm{nm}$ and the illumination spot diameter was about $20 \mu \mathrm{m}$.

To quantitatively compare the strengths of the two counterpropagating excited SPP waves, we first performed scans across the central spot. The measured maps of near-field strength (square root of field intensity) under TE and TM illuminations are shown in Fig. 7. For the TM case, a nearfield enhancement is evident and we can see waves propagating outside the central spot area to the left $(-x)$ and right $(+x)$ sides, with the left-propagating waves being a bit stron- 

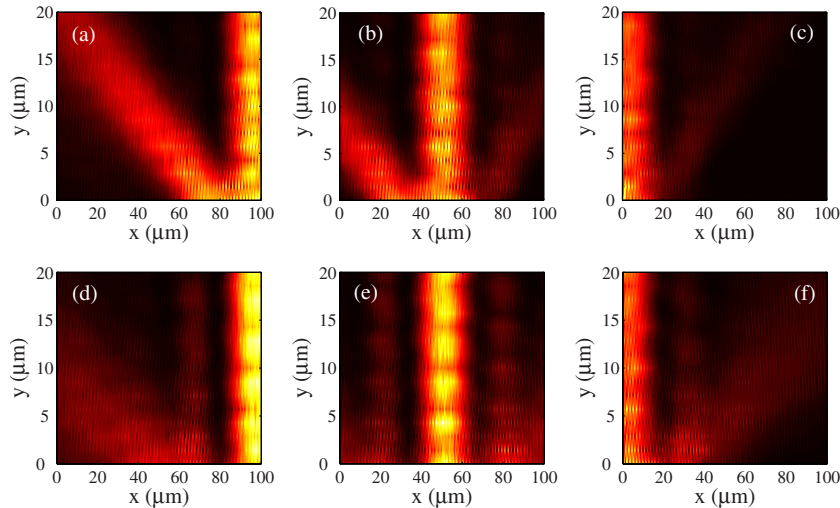

FIG. 8. (Color online) $x-y$ scan of field intensity above the air-Au interface under TM illumination. (a)-(c): scans of the left side, across the central spot, and of the right side, respectively, at $\lambda_{0}=1484 \mathrm{~nm}$. (d)-(f): the same as (a)-(c) but at $\lambda_{0}=1532 \mathrm{~nm}$. The figure color representation is the same as that in Fig. 7. Note that in this figure the origin of $y$ axis is moved to the air-metal interface for the convenience of illustration.

ger. For $\lambda_{0}<d$, the propagating \pm 1 st transmitted orders should make major contribution to the asymmetrical near field. For $\lambda_{0}>d$, however, the \pm 1 st orders become evanescent and are bound to the air-metal interface. Especially, when $\lambda_{0}$ is around $1520-1540 \mathrm{~nm}$, the phase-matching condition of SPPs is quasisatisfied and the near field should be largely contributed by the excited SPPs. We scan the field intensity in the $x y$ plane above the air-Au interface at a diffracting wavelength $\lambda_{0}=1484 \mathrm{~nm}$ as well as at a nondiffracting SPP-excitation wavelength $\lambda_{0}=1532 \mathrm{~nm}$, as shown in Fig. 8, which clearly exhibits the different characteristics of the propagating and evanescent \pm 1 st orders. Note that the numerically calculated diffraction efficiencies of the $-1 \mathrm{st}$ and +1 st transmitted orders are $16.1 \%$ and $1.6 \%$, respectively, at $\lambda_{0}=1484 \mathrm{~nm}$.

The near-field composition on a nanostructured metal surface has been thoroughly investigated by previous authors. ${ }^{14,15,18,19}$ It is found that, at near-infrared frequencies, the near field consists of not only SPPs but also a surface wave with radiative and evanescent field components (called the REC wave in the following). The REC wave creeps along the interface, whose wave number is very close to $k_{\mathrm{SPP}}$ but not exactly the same. ${ }^{14}$

We analyze the near-field composition by performing a fast Fourier transform (FFT) of the measured complex field $E(x)=A(x) \exp [i \phi(x)]$ with $A(x)$ and $\phi(x)$ as the amplitude and phase. The resolution in $k$ space is

$$
\delta k=\frac{2 \pi(N-1)}{N L},
$$

where $L$ is the scan length and $N$ is the number of sampling points. Therefore, to obtain the best resolution, we took the maximum scan length of $L=100 \mu \mathrm{m}$ and $N=512$. Then we performed the FFT analysis on the fields in the left and right sides of the spot separately in the same scan so as to compare the left- and right-propagating field strengths quantitatively. Moreover, since the central spot is so strong that the detec-
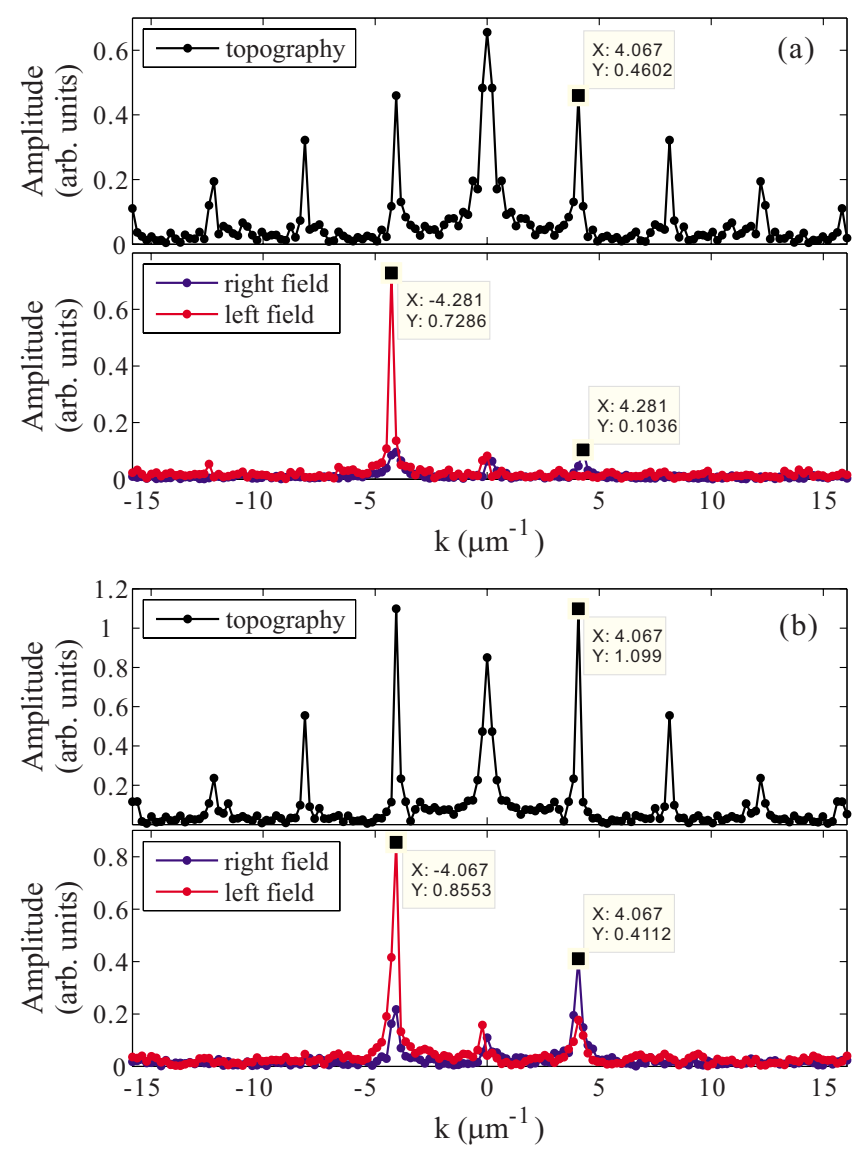

FIG. 9. (Color online) FFT analysis of the grating topography and the TM fields measured in the left and right regions indicated in Fig. 7(b), for (a) $\lambda_{0}=1484 \mathrm{~nm}$ and (b) $\lambda_{0}=1532 \mathrm{~nm}$. Several key peaks are labeled with $\mathrm{X}$ for $k$ and $\mathrm{Y}$ for the amplitude.

tion system saturates when the probe moves into the central spot area and takes time to quit the overload regime when it moves out, we exclude the central spot area and select a $30-\mu \mathrm{m}$-wide region in both the left and right sides for our analysis, as indicated in Fig. 7(b). The number of sampling points in each region is therefore 150 .

We perform the FFT analysis in the wavelength range of 1460-1580 nm. Figure 9 demonstrates two typical FFT spectra of the fields (at $\lambda_{0}=1484 \mathrm{~nm}$ and $\lambda_{0}=1532 \mathrm{~nm}$ ) as well as those of the sample topography. Note that the real topography signal produces a symmetric FFT spectrum. A main characteristic of the spectra is that for $\lambda_{0}<d$ the left/right fields always have a peak $k$ component larger than that of the topography [e.g., $k_{\text {field }}=4.281 \mu \mathrm{m}^{-1}>k_{\text {topo }}=4.067 \mu \mathrm{m}^{-1}$ in Fig. 9(a)], which should correspond to the REC wave as there is no SPP excitation in this regime. For $\lambda_{0}>d$, however, the \pm 1 st orders become evanescent and the peak $k$ value of the field FFT spectra is the same as that of the topography spectra, as shown in Fig. 9(b). This is a characteristic of the SPP excitation as the phase-matching condition [Eq. (3)] leads to $k_{\mathrm{SPP}}=k_{\text {topo }}$. In Fig. 9(b), the left-field spectrum has a dominant negative $k$ component representing a SPP wave propagating in $-x$ direction and the right field has a dominant SPP wave propagating in $+x$ direction. The strength ratio of the two peaks can to some extent reflect the 
TABLE I. Experimental and numerically calculated asymmetrical coupling ratio $r_{ \pm}$of the SPPs on the BLACES sample.

\begin{tabular}{lcccc}
\hline \hline $\begin{array}{l}\lambda_{0} \\
(\mathrm{~nm})\end{array}$ & $r_{\text {exp_fwd }}$ & $r_{\text {exp_bwd }}$ & $r_{\text {exp_mean }}$ & $r_{\text {num }}{ }^{\mathrm{a}}$ \\
\hline 1524 & 2.02 & 2.03 & 2.03 & 1.50 \\
1528 & 1.88 & 1.74 & 1.81 & 1.58 \\
1532 & 2.08 & 1.64 & 1.86 & 1.70 \\
1536 & 1.33 & 1.49 & 1.41 & 1.84 \\
1540 & 2.11 & 1.59 & 1.85 & 1.99 \\
\hline \hline
\end{tabular}

${ }^{\mathrm{a}} r_{\text {exp_fwd }}$ and $r_{\text {exp_bwd }}$ are experimental results obtained from forward and backward acquisitions, respectively, and $r_{\text {exp_mean }}$ is the mean value of them. $r_{\text {num }}$ is the numerically calculated ratio.

asymmetrical coupling effect of SPPs because in this regime the SPPs contribute the most to the near field. To best avoid the influence of the probe saturation, we have implemented both forward and backward acquisitions (i.e., the probe scans from left to right and also from right to left). The asymmetrical coupling ratio $r_{ \pm}$derived from the experimental data is listed in Table I for several wavelengths in the SPPexcitation regime, which are also compared with the numerical results. We can see certain correspondence between the experimental and theoretical ratios.

We should note that at SPP excitation the REC wave is still present. Therefore, the experimental ratio in Table I is just an estimate of the asymmetrical excitation of SPPs rather than an exact evaluation. To have a close look at the surface wave composition, we have performed an FFT analysis on the field scanned only on the left side of the spot with the best resolution ( $L=100 \mu \mathrm{m}$ and $N=512$ ), as shown in Fig. 10. The grating period derived from $k_{\text {topo }}=4.076 \mu \mathrm{m}^{-1}$ is

$$
d \pm \delta d=\frac{2 \pi}{k} \pm \frac{2 \pi}{k^{2}} \delta k=1.541 \pm 0.024 \mu \mathrm{m},
$$

which coincides with our sample design. We can see that within the main peak there are two subpeaks $k_{\text {field }}$ $=4.076 \mu \mathrm{m}^{-1}=k_{\text {topo }}$ and $k_{\text {field }}=4.327 \mu \mathrm{m}^{-1}>k_{\text {topo }} \quad$ (the

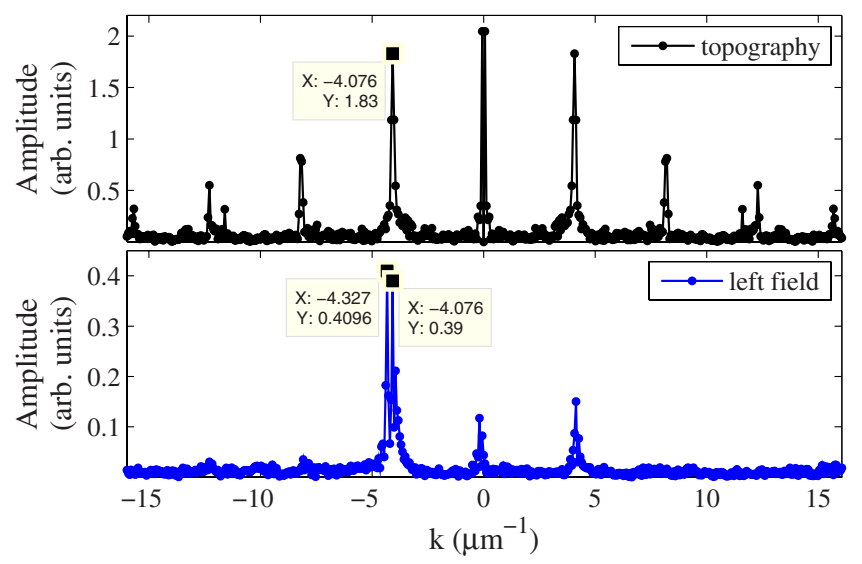

FIG. 10. (Color online) FFT analysis of the grating topography and the TM field measured on the left side of the spot with $L$ $=100 \mu \mathrm{m}, N=512$, and $\lambda_{0}=1532 \mathrm{~nm}$.

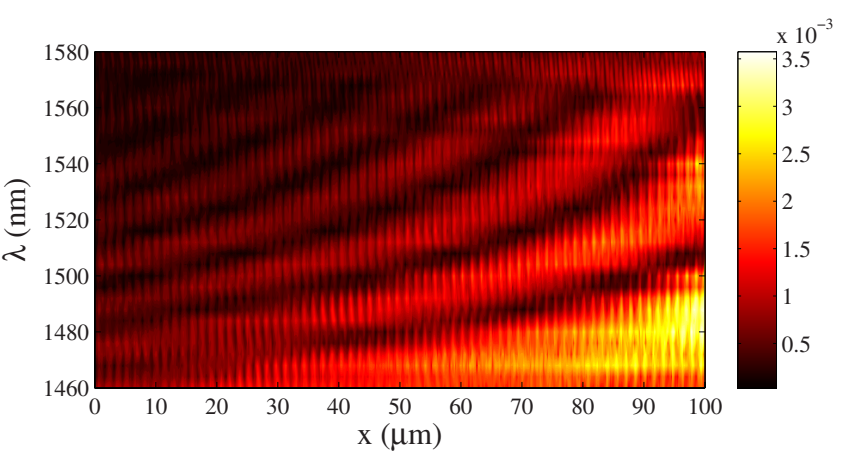

FIG. 11. (Color online) Similar scan as Fig. 7 on the left side of the spot under TM illumination. The color scale is in arbitrary units.

negative sign representing the propagation direction is omitted); the former is the SPP wave and the latter is the REC wave. It is known that when the SPPs are excited and are propagating on the surface of the grating, the SPPs are scattered by the periodic features to SPPs (primarily the backward propagating SPP wave corresponding to the small positive $k$ peak in Fig. 10) and to leakage radiation. ${ }^{11}$ This leads to the broadening of the spectral peak as well as its splitting into subpeaks. The interference of the SPP wave and the REC wave makes major contribution (as their $k$ peaks are dominant in the spectrum of Fig. 10) to the near-field interference pattern, which can be seen clearly as the main brightdark beat fringes in Fig. 11. The fringe spacing calculated from the beat frequency $\Delta k=4.327-4.076=0.251 \mu \mathrm{m}^{-1}$ is $25 \mu \mathrm{m}$ at $\lambda_{0}=1532 \mathrm{~nm}$, which is consistent with that observed in Fig. 11. The other small coarse structures in the near-field pattern of Fig. 11, such as the additional modulation around $\lambda_{0}=1540 \mathrm{~nm}$, may be ascribed to other interference processes between the surface waves (the field components corresponding to other minor $k$ values within the main $k$ peak in Fig. 10) such as the interference of SPP waves scattered by the triangular surface features to propagate in different directions. These minor interactions are more complicated and are nevertheless out of the scope of current study.

\section{Concluding remarks}

We have demonstrated in theory that the BLACES can achieve large asymmetrical or even unidirectional coupling of SPPs on a dielectric-metal interface at normal incidence, just as observed with classical 1D slanted gratings. An advantage of this kind of binary-blazed grating is that they can be easily fabricated by standard nanofabrication methods such as EBL and lift-off technique, which are more favorable in applications.

Then, through a preliminary experiment, we have characterized by near-field microscopy the asymmetrical coupling effect of SPPs on a BLACES sample, and have found satisfactory agreement between theoretical and experimental results. Since the effect is relatively small due to some practical restrictions in our current experiment, it is just a first corroboration of our theoretical prediction and needs to be further optimized. 

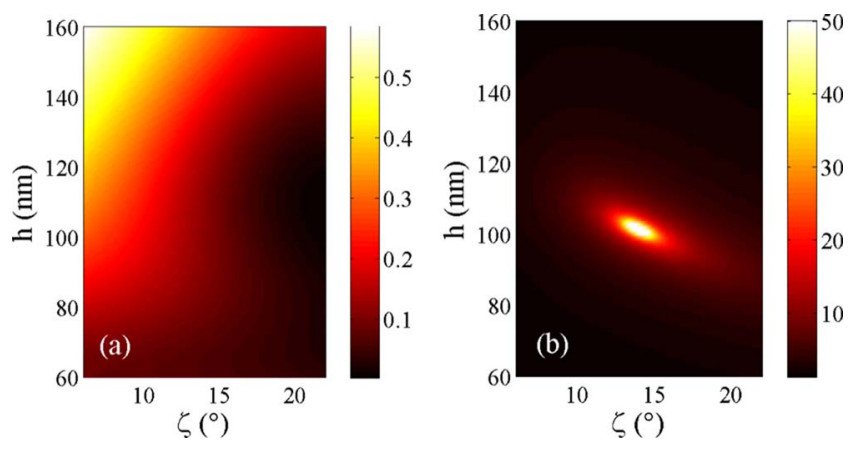

FIG. 12. (Color online) Optimization of the slanted grating with $d=1057.9 \mathrm{~nm}$. (a) Diffraction efficiency of the zeroth reflected order; (b) amplitude ratio $r_{ \pm}$(in the central white region, $r_{ \pm}>50$ ).

Comparing Figs. 2 and 6, we can see that a key factor in achieving large asymmetrical coupling effect of SPPs is to best suppress the strength of the +1 st order (ideally to null) while the -1 st order is enhanced at SPP excitation. According to our numerical experience, whether the +1 st order is enhanced or suppressed is affected by many factors such as structural parameters, illumination condition, and material properties. A comprehensive study on the influence of these factors on the SPPs coupling should be made. So far, we have not found a simple principle to guide the structural configuration except for time-consuming numerical optimization. Therefore, it is necessary to understand the physics of how the \pm 1 st evanescent orders are enhanced or suppressed, which will be the task of our future study.

\section{CLASSICAL 1D SLANTED GRATING}

\section{A. Sample design and fabrication}

As a comparative study, we also launched an experimental investigation of the asymmetrical coupling effect of SPPs on 1D slanted gratings. For this purpose, we designed an aluminum slanted sinusoidal grating with the geometry shown in Fig. 1(b). The light source is chosen with the following considerations. On the one hand, the charge-coupled device (CCD) camera used in the characterization setup (shown later) is sensitive to visible and near-infrared light, and the wavelength should not be too long for the effective excitation of SPPs. On the other hand, the SPPs are expected to propagate on the grating surface as long as possible so as to facilitate the imaging of the SPP tails. It requires the low loss of surface waves during propagation, which is satisfied at longer wavelengths. Therefore, as a compromise between the two considerations, the wavelength is selected as $\lambda_{0}$ $=1064 \mathrm{~nm}$, at which the refractive index of aluminum is $n_{\text {met }}=1.2190+i 10.4161$. The cover medium is air.

We used the Chandezon method, ${ }^{20}$ which is most suitable for smooth profile gratings, to perform the rigorous simulations. With an initial estimate of $d \approx 1059.3 \mathrm{~nm}$ derived from Eqs. (1) and (3), the grating period is numerically optimized to $d=1057.9 \mathrm{~nm}$. Then the other two structural parameters, the inclination angle $\zeta$ and the groove depth $h$, are optimized as shown in Fig. 12. With the optimal parameters $\zeta=13.8^{\circ}$ and $h=102 \mathrm{~nm}$, the diffraction efficiency of the ze-
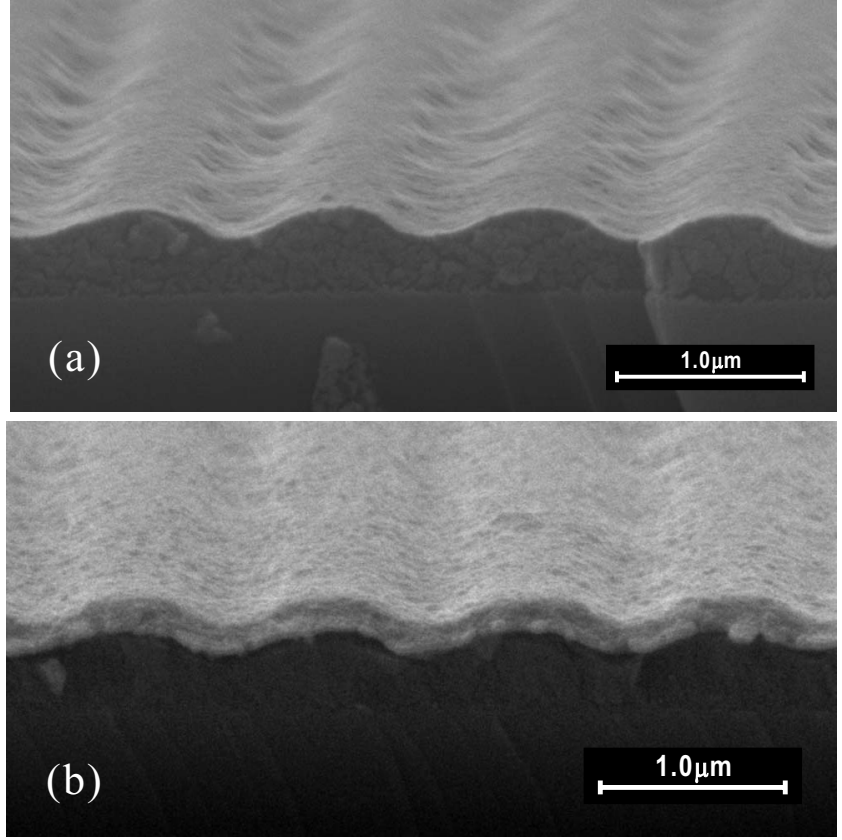

FIG. 13. SEM images of (a) a slanted sinusoidal photoresist grating and (b) the final grating after $\mathrm{Al}$ deposition.

roth reflected order can be suppressed to $12 \%$ and the ratio of the \pm 1 st-order amplitudes is maximized to $r_{ \pm}=185$, in which case most of the incident energy should be coupled via the -1 st order to the SPP wave propagating in the $-x$ direction. The optimization results are comparable with those obtained by Bonod et al. ${ }^{6}$ However, it is seen that the optimized structure is a slightly slanted shallow-groove grating and the tolerance of structural parameters to achieve large $r_{ \pm}$is quite small, which imposes a major challenge in fabrication to precisely realize the designed profile.

We employed single-beam holographic lithography ${ }^{21}$ to fabricate the slanted sinusoidal grating. First, a 450-nm-thick layer of Shipley 9912 antireflection positive photoresist was spin coated on a thick glass substrate. Then the resist was pre-exposed under the uniform illumination of an expanded $441.6 \mathrm{~nm} \mathrm{He}-\mathrm{Cd}$ laser beam to weaken its development nonlinearity. To create the slanted sinusoidal profile, the resist layer was then exposed to an interference pattern generated by a Lloyd-mirror interferometer ${ }^{21}$ with asymmetrical recording geometry, in which the sample normal was tilted by an angle with respect to the bisector of the two exposing $\mathrm{He}-\mathrm{Cd}$ laser beams (the inclination angle $\zeta$ of the slanted grating is thus controlled by this tilt angle and the angle between the two exposing beams). Afterward, the resist was developed with Shipley 351 developer. Finally, a thick Al layer (about $160 \mathrm{~nm}$ thick to ensure no light transmission) was deposited onto the surface-relief resist grating by thermal evaporation. The resulting grating can therefore be regarded as an optically infinitely thick Al grating with slanted sinusoidal profile as designed.

Figure 13 shows the SEM pictures of a fabricated sample before and after $\mathrm{Al}$ deposition. The structural parameters are $d=1058 \mathrm{~nm}, h=152 \mathrm{~nm}$, and $\zeta=13.7^{\circ}$. Obviously, the groove height $h$ is too large compared with the optimal design. This is due to the strong nonlinearity of the resist in 


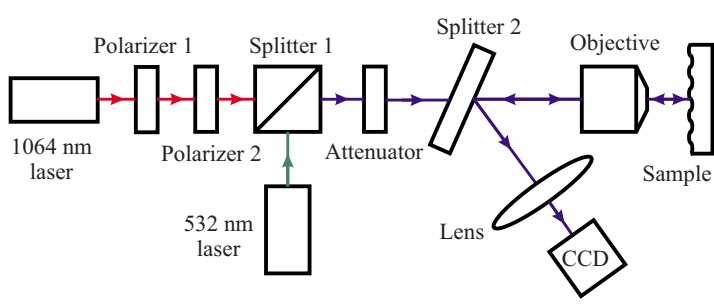

FIG. 14. (Color online) Sketch of the SPPs far-field imaging system.

development. We have made considerable trials by changing, e.g., exposure dose and development conditions, but unfortunately still failed to get satisfactory shallow profiles. We found that, when $h$ approaches $100 \mathrm{~nm}$, the impact of development nonlinearity is greater and the top of the grating ridges is almost flat.

\section{B. Far-field characterization}

We characterized the excitated SPPs by far-field imaging. As mentioned before, since the SPPs can be scattered to leakage radiation when they are propagating on the periodic surface of the grating, it is possible to visualize the SPPs by imaging the far-field radiation. ${ }^{2,22}$

The characterization setup is illustrated in Fig. 14. A 1064 $\mathrm{nm}$ laser beam passing through two linear polarizers is tuned as TE or TM polarized with fixed intensity, which is then combined with a $532 \mathrm{~nm}$ laser beam via a beam splitter. The green laser acts as a visual guide to facilitate the alignment of the optical system. After attenuation, the laser beam passes through another beam splitter and a microscope objective (with magnification $40 \times$ and numerical aperture 0.66 ), and illuminates the surface of the $\mathrm{Al}$ grating. The leakage radiation emitted by the SPPs propagating on the grating surface is collected by the objective and imaged onto a CCD camera via the microscopic imaging system consisting of the objective, beam splitter 2, a lens, and the CCD.

Figure 15 shows the microscopic images of the propagating SPPs on a grating sample under TM and TE illumina-

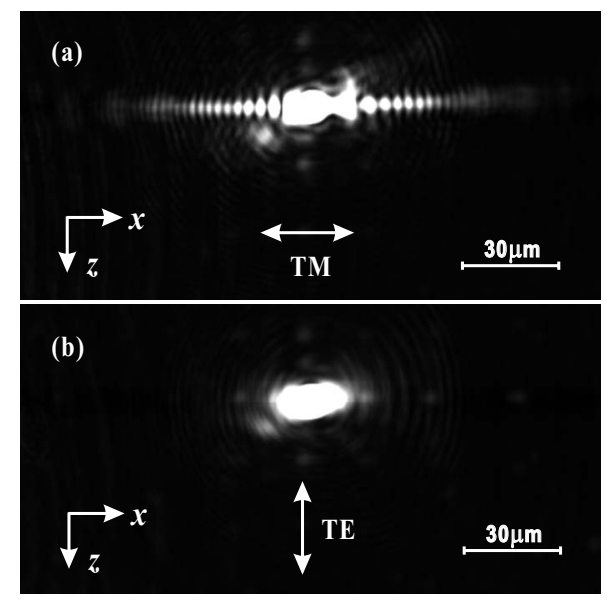

FIG. 15. Far-field images of the excited SPPs on an Al slanted grating sample under (a) TM and (b) TE illuminations. tions. The excitation of SPPs by TM-polarized light is evident. In Fig. 15(a), we can see two asymmetrical tails on the left and right sides of the central spot, reflecting the asymmetrically excited SPP waves propagating in $\pm x$ directions. The left tail is longer, indicating that the SPP excitation on this side is stronger. Note that the fringes in the tails are probably caused by the interference of the SPP field with the diffraction rings of the objective aperture because under TE illumination these fringes are still visible although very faint.

To quantitatively compare the experimental results with the theoretical ones, we need to calculate the asymmetrical coupling ratio $r_{ \pm}$of the SPPs. It is known that the electric field of the SPPs propagating in $\pm x$ directions on the metal surface can be expressed as ${ }^{2}$

$$
E_{\mathrm{SPP}}^{ \pm 1}(x)=E_{0}^{ \pm 1} \exp \left( \pm i \alpha x \mp \alpha^{\prime} x\right),
$$

where $E_{0}^{ \pm 1}$ are the amplitudes of the two SPP waves, and $\alpha$ and $\alpha^{\prime}$ are the real and imaginary parts of the SPP complex propagation constant $k_{\mathrm{SPP}}$, respectively. Note that the two counterpropagating SPP waves have one and the same propagation constant $k_{\mathrm{SPP}}$ despite the asymmetrical profile, and the asymmetrical coupling strengths are caused only by the different field amplitudes $E_{0}^{ \pm 1}{ }^{6}$ Since the detected leakage radiation from a surface point is proportional to the local SPP field intensity, the detected radiation intensity observes the relation

$$
I^{ \pm 1}(x) \propto\left|E_{\mathrm{SPP}}^{ \pm 1}(x)\right|^{2}=\left|E_{0}^{ \pm 1}\right|^{2} \exp \left(\mp 2 \alpha^{\prime} x\right) .
$$

We denote the propagation lengths of the left and right tails as $l_{-}$and $l_{+}$, respectively. Then the ends of the two tails, which are determined by the detectable minimum signal on the $\mathrm{CCD}$, should have the same intensity, i.e., $I^{-1}\left(-l_{-}\right)$ $=I^{+1}\left(l_{+}\right)$. Therefore, we can derive from Eq. (11) the asymmetrical coupling ratio as

$$
r_{ \pm} \equiv\left|E_{0}^{-1}\right| /\left|E_{0}^{+1}\right|=\exp \left[\alpha^{\prime}\left(l_{-}-l_{+}\right)\right] .
$$

Now we retrieve $\alpha^{\prime}$ and $l_{+}$from the experimental data. In order to reduce the influence of the slight deviation of the incident beam from the normal direction, we performed two measurements on the same sample, one with the sample mounted as depicted in Fig. 1(b) and the other with the sample rotated by $180^{\circ}$ about the $y$ axis (i.e., the $\pm x$ directions are reversed). Figure 16 shows the far-field images of the SPP tails measured on our best sample [the one shown in Fig. 13(b)]. To calculate $\alpha^{\prime}$ and $l_{+}$, we first obtained pixel coordinates and gray values of the bright speckles in each tail, and then used an exponential fit to the data, as shown in Fig. 17, to obtain the decay coefficient $\alpha^{\prime}$. The tail-length difference $l_{-}-l_{+}$is obtained from the pixel coordinates. Note that in imaging the tails in Fig. 16 we increased the attenuation of the incident light so that the bright speckles of the tail images are not saturated on the CCD pixels and therefore enable the exponential fit to them.

Table II summarizes the retrieved parameters from the two measurements. The derived asymmetrical SPP coupling ratio $r_{ \pm}$is 1.44 , which is comparable with the theoretical value of 1.90 that we calculated numerically with the practical structural parameters of the sample. To compare the experimental $\alpha^{\prime}$ value with the theoretical one, we calibrated 


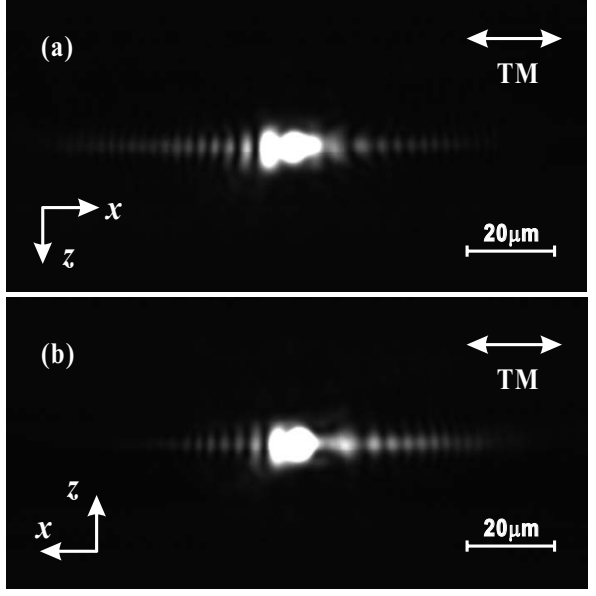

FIG. 16. Far-field imaging of the SPP tails on the same sample mounted (a) as in Fig. 1(b) and (b) with the $\pm x$ directions reversed.

the pixel coordinate as $0.294 \mu \mathrm{m} /$ pixel by imaging a $10 \mu \mathrm{m}$ standard scale. Then the experimental $\alpha^{\prime}$ value derived from Table II is $0.03543 \mu \mathrm{m}^{-1}$, which is also in good correspondence with the numerically calculated theoretical value of $0.03341 \mu^{-1}$. The discrepancies may be caused by factors such as the distortion of the sinusoidal grating profile, the measurement uncertainty of the structural parameters, and the errors in far-field imaging system.

\section{Concluding remarks}

We have designed and fabricated slanted sinusoidal Al gratings and have characterized, by far-field imaging, the asymmetrical coupling of SPPs on the grating surface at normal incidence. Good agreement between theory and experiment has been obtained, which corroborates the previous theoretical prediction that the blazed grating can lead to asymmetrical coupling of SPPs. ${ }^{6}$

However, the realized asymmetrical coupling effect is still rather weak compared with the optimal design, which is restricted by technical difficulties in fabrication. Further efforts need to be made to overcome the problems in generating the very-shallow-groove slightly slanted sinusoidal profiles. The optimization of the grating structure is also aided if the struc-

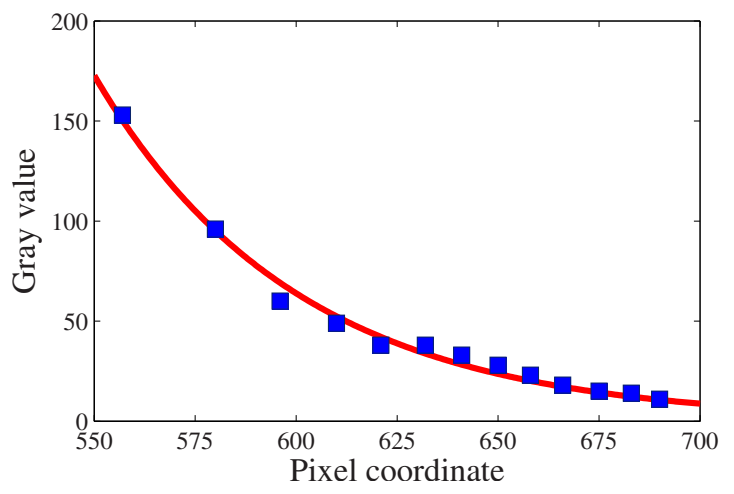

FIG. 17. (Color online) Exponential fit to a measured right tail.
TABLE II. Parameters retrieved from the far-field imaging data of the SPP tails on the slanted grating sample.

\begin{tabular}{llccc}
\hline \hline & & Meas. 1 & Meas. 2 & Mean \\
\hline$\alpha^{\prime}$ & From left tail & 0.00984 & 0.00981 & \\
$(1 /$ pixel $)$ & From right tail & 0.00995 & 0.01205 & \\
& Mean value & 0.00990 & 0.01093 & 0.01042 \\
$l_{-} l_{+}$(pixel) & 29 & 40 & 34.5 \\
$r_{ \pm}$calculated by Eq. (12) & 1.33 & 1.55 & 1.44 \\
\hline \hline
\end{tabular}

${ }^{a}$ Meas. 1 and 2 refer to two measurements by mounting the sample as in Fig. 1(b) and with the $\pm x$ directions reversed, respectively.

tural parameter tolerance can be loosened so as to facilitate fabrication.

\section{CONCLUSIONS}

In this work, we have investigated both theoretically and experimentally the asymmetrical coupling effect of SPPs on two types of blazed gratings at normal incidence.

First, we proposed the use of a 2D blazed grating, the BLACES, as an alternative to the classical 1D slanted gratings to achieve the asymmetrical coupling effect. The advantage is that the binary structures can be fabricated with mature standard nanofabrication methods such as EBL and liftoff technique. Numerical simulations demonstrated the ability of the BLACES to produce largely asymmetrical or even unidirectional excitation of SPPs, just as the 1D slanted gratings can do.

Second, we fabricated BLACES samples and launched a preliminary experiment to characterize the asymmetrical coupling of SPPs by multiheterodyne near-field microscopy. Even though the realized effect is relatively small due to some limitations in our experiment, good agreement between experimental and theoretical results has been obtained and the near-field properties of the excited SPP waves were also analyzed.

Third, as a comparative study, we also investigated experimentally the asymmetrical coupling effect of SPPs on classical 1D slanted gratings and compare it with numerical simulation. A batch of slanted sinusoidal Al gratings were fabricated and characterized by far-field imaging of the leakage radiation of the scattered SPPs. The good agreement between theory and experiment corroborates the previous theoretical prediction. However, the asymmetrical coupling effect is still rather weak due to the fabrication difficulty in realizing the very-shallow-groove slightly slanted sinusoidal grating profile.

In conclusion, both blazed gratings have been shown to be able to excite SPPs asymmetrically at normal incidence as predicted theoretically. Both the near-field microscopy and the far-field imaging method are feasible to characterize the asymmetrical coupling effect of SPPs.

Further study needs to be made to improve the performance of the elements, such as suppressing the $+1 \mathrm{st}(-1 \mathrm{st})$ order while enhancing the $-1 \mathrm{st}(+1 \mathrm{st})$ order at SPP excitation, designing optimized structures that are well adapted to the 
characterization method, and loosening the structural parameter tolerance to facilitate fabrication. These will rely on more insight into the physics of the effect as well as the improvement of the experimental techniques. Furthermore, to make the elements competent for applications in such as integrated photonic circuits, the coupling efficiency of the incident light to SPPs should be maximized in addition to the optimization of the asymmetrical coupling effect.

\section{ACKNOWLEDGMENTS}

We acknowledge the support by the Academy of Finland (Contracts No. 128420, No. 129155, and No. 209806), the China Scholarship Council, the Swiss National Science Foundation, and the Network of Excellence in Micro-optics (NEMO) (www.micro-optics.org).
*Corresponding author; bai@joyx.joensuu.fi

${ }^{1}$ W. L. Barnes, A. Dereux, and T. W. Ebbesen, Nature (London) 424, 824 (2003).

${ }^{2}$ S. A. Maier, Plasmonics: Fundamentals and Applications (Springer, New York, 2007).

${ }^{3}$ D. Egorov, B. S. Dennis, G. Blumberg, and M. I. Haftel, Phys. Rev. B 70, 033404 (2004).

${ }^{4}$ J.-Y. Laluet, E. Devaux, C. Genet, T. W. Ebbesen, J.-C. Weeber, and A. Dereux, Opt. Express 15, 3488 (2007).

${ }^{5}$ F. López-Tejeira, S. G. Rodrigo, L. Martín-Moreno, F. J. GarcíaVidal, E. Devaux, T. W. Ebbesen, J. R. Krenn, I. P. Radko, S. I. Bozhevolnyi, M. U. González, J. C. Weeber, and A. Dereux, Nat. Phys. 3, 324 (2007).

${ }^{6}$ N. Bonod, E. Popov, L. Li, and B. Chernov, Opt. Express 15, 11427 (2007).

${ }^{7}$ Diffractive Optics for Industrial and Commercial Applications, edited by J. Turunen and F. Wyrowski (Akademie Verlag, Berlin, 1997).

${ }^{8}$ B. H. Kleemann, J. Ruoff, and R. Arnold, Opt. Lett. 30, 1617 (2005).

${ }^{9}$ P. Lalanne, S. Astilean, P. Chavel, E. Cambril, and H. Launois, Opt. Lett. 23, 1081 (1998).
${ }^{10}$ H. Elfström, M. Kuittinen, T. Vallius, B. H. Kleemann, J. Ruoff, and R. Arnold, Opt. Commun. 266, 697 (2006).

${ }^{11}$ A. V. Zayats, and I. I. Smolyaninov, J. Opt. A: Pure Appl. Opt. 5, S16 (2003).

${ }^{12}$ B. Bai and L. Li, J. Opt. A: Pure Appl. Opt. 7, 271 (2005).

${ }^{13}$ A. Lehmuskero, M. Kuittinen, and P. Vahimaa, Opt. Express 15, 10744 (2007).

${ }^{14}$ P. Lalanne and J. P. Hugonin, Nat. Phys. 2, 551 (2006).

${ }^{15}$ L. Chen, J. T. Robinson, and M. Lipson, Opt. Express 14, 12629 (2006).

${ }^{16}$ U. Schröter and D. Heitmann, Phys. Rev. B 60, 4992 (1999).

${ }^{17}$ E. Descrovi, T. Sfez, L. Dominici, W. Nakagawa, F. Michelotti, F. Giorgis, and H.-P. Herzig, Opt. Express 16, 5453 (2008).

${ }^{18}$ L. Aigouy, P. Lalanne, J. P. Hugonin, G. Julié, V. Mathet, and M. Mortier, Phys. Rev. Lett. 98, 153902 (2007).

${ }^{19}$ H. Liu and P. Lalanne, Nature (London) 452, 728 (2008).

${ }^{20}$ L. Li, J. Opt. Soc. Am. A Opt. Image Sci. Vis. 16, 2521 (1999).

${ }^{21}$ X. Mai, R. Moshrefzadeh, U. J. Gibson, G. I. Stegeman, and C. T. Seaton, Appl. Opt. 24, 3155 (1985).

${ }^{22}$ A. Hohenau, J. R. Krenn, A. L. Stepanov, A. Drezet, H. Ditlbacher, B. Steinberger, A. Leitner, and F. R. Aussenegg, Opt. Lett. 30, 893 (2005). 